\title{
Active steering control-based trucks lane-keeping system
}

\section{Kawther Osman*}

National School of Engineering of Sousse (ENISO), CEM Lab ENIS,

Sousse, Tunisia

Email: osmankawthar22@gmail.com

*Corresponding author

\section{Jawhar Ghommam}

Department of Electrical and Computer Engineering,

Sultan Quaboos University,

Muscut, Oman

Email: jawher@squ.edu.om

\section{Maarouf Saad}

Département de Génie Électrique,

École de Technologie Supérieure,

Montréal, Canada

Email: maarouf.saad@etsmtl.ca

\begin{abstract}
This paper proposes a lane-keeping system for trucks with trailer. The chosen active steering vehicle model allows to control both steering angles of the vehicle's articulations to pursue a desired trajectory. Thus, a guidance module is first implemented to define a bounded path parameter to follow. This module takes advantage of the Barrier Lyapunov function to impose constraints on the truck's longitudinal and lateral position and the trailer's orientation w.r.t the truck namely the articulation angle. The applied constraints guarantee a safe lane keeping for such type of vehicle while restricting the vehicle position inside the lane boundaries. The bounded path parameter is thereafter followed by a Robust Integral of Sign of Error (RISE) feedback controller. The proposed controller calculates the appropriate truck and trailer's steering angles to ensure the safe lane keeping and compensates the vehicle model uncertainties.
\end{abstract}

Keywords: articulated truck; lane keeping; path following; robust controller; LOS guidance; Barrier Lyapunov function.

Reference to this paper should be made as follows: Osman, K., Ghommam, J. and Saad, M. (2021) 'Active steering control-based trucks lane-keeping system', Int. J. Cybernetics and Cyber-Physical Systems, Vol. 1, No. 1, pp.33-51.

Biographical notes: Kawther Osman received her MS degree in Computer and Control Engineering from the National Institute of Applied Sciences and Technology (INSAT), University of Carthage, Tunis, Tunisia in 2015. Currently, she is pursuing the PhD degree with the National School of 
Engineering of Sousse, University of Sousse, and the Control and Energy Management Laboratory (CEM-Lab) in Tunisia. Her current research interests include intelligent transportation systems, non-linear control, vehicle navigation and vision-based road perception.

Jawhar Ghommam received his BSc degree in Computer and Control Engineering from the INSAT in 2003 in Tunis. He got the DEA (MSc) degree from the University of Montpelier at the Laboratoire d'Informatique, Robotique et Micro-électronique (LIRMM, France) in 2004 and later on in 2008 his $\mathrm{PhD}$ degree in Control Engineering jointly from the National Engineering School of Sfax and the University of Orleans in France. He is an Associate Professor of Control Engineering at Sultan Quaboos University (SQU) in Oman and occupies a Permanent Senior Research Position at the CEM lab, Enis, Sfax, Tunisia. His research interests include fundamental motion control concepts for non-holonomic/underactuated vehicle systems, intelligent and autonomous control of networked unmanned systems.

Maarouf Saad received his BSc and MSc degrees in Electrical Engineering from École Polytechnique of Montreal, Montreal, Quebec, Canada in 1982 and 1984, respectively. He got the $\mathrm{PhD}$ degree in Electrical Engineering from McGill University, Montreal, Quebec, Canada in 1988. He joined École de Technologie Supérieure in 1987 where he is teaching Control Theory and Robotics Courses. His research interests include non-linear control and optimisation applied to robotics and flight control system, rehabilitation robotics, power systems and distributed generation.

This article is a revised and expanded version of a paper entitled 'Robust Active Steering Control for Articulated Vehicle' presented at the 'International Multi-Conference on Systems, Signals and Devices 2020'.

\section{Introduction}

Recent decades witnessed an outstanding evolution of the transportation system's automation. Lane-keeping systems are among the challenging ones. They may be applicable to simple or articulated vehicle models.

Obviously, additional articulation leads to complex models so a tougher way to control it and to avoid the instability phenomena is required. A good understanding of the source of the instability helps to reduce its consequences. By focusing only on the movements on the yaw plan, one may distinguish two types of instability due to the swaying and the jackknifing. The first one is caused by oscillatory motion of the vehicle position which may result in a deviation from its main trajectory. The second instability type is due to the increased oscillations of the articulation angle separating the truck from its trailer. The main factor affecting this instability is the driver's loss of the control. A way to resolve this problem is to assist the driving manoeuvre, by warning the driver whenever he lose control and deviates from the safe desired trajectory, or to automate the whole lane-keeping manoeuvre using controllers.

This paper proposes a novel approach to ensure an automatic constrained lane keeping for articulated vehicles. This approach is composed of two main modules. The first module imposes constraints on the truck's position to avoid the swaying phenomena and limit the trailer's orientation to prevent the jackknifing of the trailer. The second 
module ensures the control of the steering angles of both vehicle's articulations to follow a desired trajectory safely.

\subsection{State of the art}

The authors in Pillajo-Quijia et al. (2020) analysed and classified the factors of road accidents for trucks using machine learning to further improve road driving safety. One way to make driving safer is to support the lane keeping for heavy trucks with a tactile feedback in Montiglio et al. (2006). The proposed system generates an additional steering torque to avoid lane departure. On another way, Zhang et al. (2019) developed a sliding mode based steering and braking control, that calculates the appropriate lateral force and yaw moment for each wheel of the multi-axle truck. An optimisation stage is implemented to decrease the computation time. In Miao and Cebon (2019), a PID active trailer steering controller is studied for tractor-semitrailer, B-double and A-triple vehicle models. The authors studied the effect of vehicle speed and road camber and sliding surface on the vehicle's stability. The aforementioned conditions reduce the precision of the vehicle position and hence give erroneous trailer steering angles. Zhu et al. (2019) studied the offered robustness of different active steering trailer controllers under huge lateral accelerations. The simulation results show that the Mu-Synthesis (MS) is the most robust to uncertainties caused by the mass or the longitudinal position of the trailer. Apart from the yaw stability discussed in the aforementioned paper, the roll stability present an important safety factor, in Sename et al. (2019) a linear parameter varying controller allows to reduce the rollover effect using the TruckSim ${ }^{C}$ vehicle model. Otherwise, the developed approach in Nilsson (2013) combines vehicle and environment information to estimate a desired lateral position and define a guiding force to fulfil the lane-keeping task, the authors used an adaptive controller to steer the vehicle accordingly. In Zhang et al. (2020), the use of exclusive lanes improves the longitudinal and the lateral safety for trucks. In fact, the authors compared two controllers in path tracking manoeuvre, the sliding mode controller allows a better performance than the integral-plus-state feedback controller. In Liu et al. (2020), a lateral controller is proposed to perform a robust lane keeping against the external disturbances. The developed system is based on an adaptive state estimator and a finite time optimised state feedback regulator. Chen et al. (2019) studied the effects of lateral perturbation of automated trucks using finite element analysis. All the aforementioned papers considered the truck's lateral control. Otherwise, Shi et al. (2019) proposed a trajectory and velocity planner that avoids collision and rollover scenarios for autonomous trucks.

In Hac et al. (2008), two active trailer braking approaches are developed to control the truck's yaw dynamics. However, in Chen and Tomizuka (2000), the coordinated steering angle and braking of the trailer are laterally controlled using the backstepping technique. Keymasi-Khalaji et al. (2015) developed a path tracking controller for a tractor with two trailers using a non-model-based PD controller. In Islam et al. (2012), the truck's steering angle is guided using a PID controller. Meanwhile, the Linear Quadratic Regulator is used to control the trailer's front steering wheel. In Khalaji et al. (2019), a Robust Adaptive Look-Ahead Kinematic Controller is proposed to compensate the vehicle model's uncertainties and to follow a desired path. The authors use in addition an Adaptive Computed torque to stabilise the whole system. Kassaeiyan et al. (2019) combined Linear and Non-linear Model Predictive Controllers to avoid the selfcollision and to track a desired trajectory. In Yue et al. (2019), the truck's parametric 
perturbations are identified and compensated using the radial basis function neural network and the sliding mode controller respectively. In Xu et al. (2019), an optimal performance index and a shift register algorithm are conceived to follow a reference trajectory and to guarantee the vehicle's lateral stability. In Shamim et al. (2011), the active trailer steering and braking controllers are studied along with the variable geometry approach to define the articulated vehicle's lateral stability. Most of the previous works focused on the active trailer front wheel control, however, a Compliant Steering system is proposed in Qingyun et al. (2017) to act on the rear active trailer wheel while ensuring its lateral stability.

To model the truck with trailer dynamics, some works adopted the front active trailer wheel, the other considered the active rear trailer wheel, while others concerned the passive trailer steering wheel. The chosen model is thereafter used to develop either an active or a passive trailer steering controller in order to follow a desired trajectory by the articulated vehicle. It is noted that the articulated vehicle's control is a very challenging research field due to the unstable motions of the trailer. One way to overcome this problem is to impose constraints on the articulated vehicle's position and orientation while ensuring the lane keeping.

\subsection{Contribution}

In order to follow a desired trajectory and avoid the skidding of a truck-trailer combination, a Line of Sight (LOS) guidance system along with a RISE controller is proposed. An active trailer steering vehicle model is adopted to control the orientation of both the truck and its trailer. The guidance system constrains the positions of the vehicle inside the lane. In fact, the longitudinal and the lateral truck COG position are bounded by a desired longitudinal position and the lane boundaries respectively. Otherwise, the articulation angle between the truck and its trailer is limited inside a predefined safe interval. Hence, the limitation of the truck's COG position and the articulation angle allows to limit the position of the trailer's COG. This safe zone prevents the vehicle from the jackknifing and the swaying movements. The guidance system is based on the logarithm Barrier Lyapunov function which imposes hard constraints on both truck and trailer positions in the yaw plane and outputs desired path parameters and vehicle orientation. The RISE controller is thereafter used to follow the desired path parameter on one hand and compensate the vehicle parameters uncertainties on another hand, i.e. the trailer mass and its inertia.

This paper is divided into five sections: Section 2 presents the articulated vehicle dynamic model. Section 3 consists of designing a LOS guidance algorithm and a RISE feedback controller that allows a constrained lane keeping. Section 4 illustrates the numerical simulation of the developed approach along with a comparative study with the computed torque controller. The paper is concluded in Section 5.

\subsection{Problem statement}

The ultimate objective of this work is to guarantee a safe and a robust lane keeping for trucks with trailer. As the truck's position and the articulation angle with respect to the trailer are well bounded, one may be sure that the articulated vehicle is following a desired trajectory safely. Hence, the goal is to steer both the vehicle's articulations while constraining the lateral and the longitudinal positions in the first place and the 
articulation angle the a second place. Therefore, the truck's COG position $\left[x_{1}, y_{1}\right]$ should follow the desired path parameter $\left[x_{1 d}(\theta), y_{1 d}(\theta)\right]$. Thus, the tracking longitudinal and lateral errors $e$ and $s$ must converge to zero. In addition, the articulation angle $\phi$ must converge to the desired $\phi_{d}$ to ensure that the trailer's COG follows the truck's COG directly and the desired path parameter indirectly. Applying hard constraints on the position errors $e$ and $s$, and the articulation error $q=\phi_{d}-\phi$ guarantees the nonviolation of the lane envelop while proceeding the lane keeping task. The aforementioned errors are shown in the Figure 2. In addition, a robust controller is used to compensate the vehicle model's uncertainties. The whole system is presented in Figure 1.

Figure 1 Trailed truck's lane keeping bloc diagram

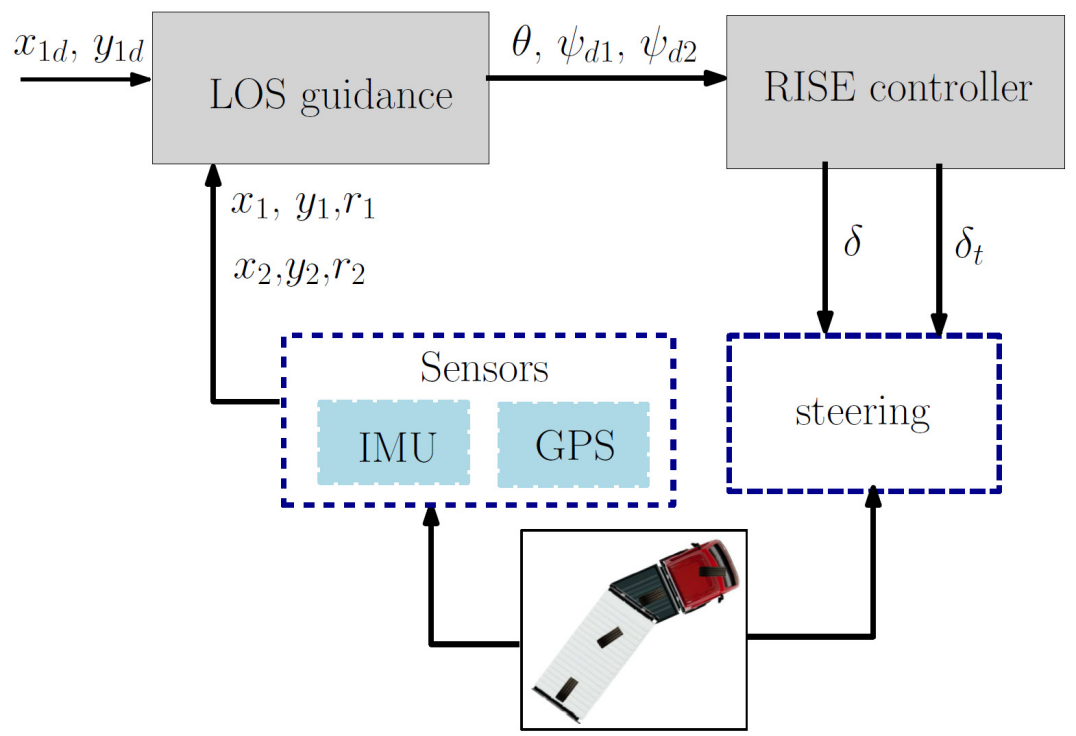

\section{Dynamic model}

In this paper, a dynamic truck with trailer model is adopted to simulate the articulated vehicle's position and motions. A few assumptions are considered to simplify this vehicle modelling. In fact, the right and the left wheels on the same axes will be assumed as one wheel which leads to a bicycle double track model, also the sideslip angle is not considered in this work. The vehicle's position is defined from a geometrical relationship as presented in Figure 2. However, the dynamic parameters are parameterised using the Newton dynamic laws.

$$
\begin{aligned}
& \dot{x}_{1}=U_{1} \cos \left(\psi_{1}\right)-V_{1} \sin \left(\psi_{1}\right) \\
& \dot{y}_{1}=U_{1} \sin \left(\psi_{1}\right)+V_{1} \cos \left(\psi_{1}\right) \\
& \dot{\psi}_{1}=r_{1} \\
& \dot{\psi}_{2}=r_{2}
\end{aligned}
$$


where $\left[x_{1}, y_{2}\right]$ denotes the position of the truck's COG, $U_{1}$ and $V_{1}$ are the longitudinal and the lateral truck velocities, $\psi_{1}$ and $\psi_{2}$ are the truck and the trailer heading angle, respectively, while $r_{1}$ and $r_{2}$ present the truck's and trailer's yaw rates. The truck's dynamics are expressed as follows:

$$
\begin{aligned}
& \dot{U}_{1}=\frac{1}{m_{1}}\left\{F_{x}-F_{y 1} \sin (\delta)+V_{1} r_{1}\right\} \\
& \dot{V}_{1}=\frac{1}{m_{1}}\left\{-F_{y}+F_{y 1} \cos (\delta)+F_{y 2}-U_{1} r_{1}\right\} \\
& \dot{r}_{1}=\frac{1}{I_{1}}\left\{F_{y} d+F_{y 1} S_{1} \cos (\delta)-F_{y 2} S_{2}\right\}
\end{aligned}
$$

Figure 2 Vehicle's applied forces

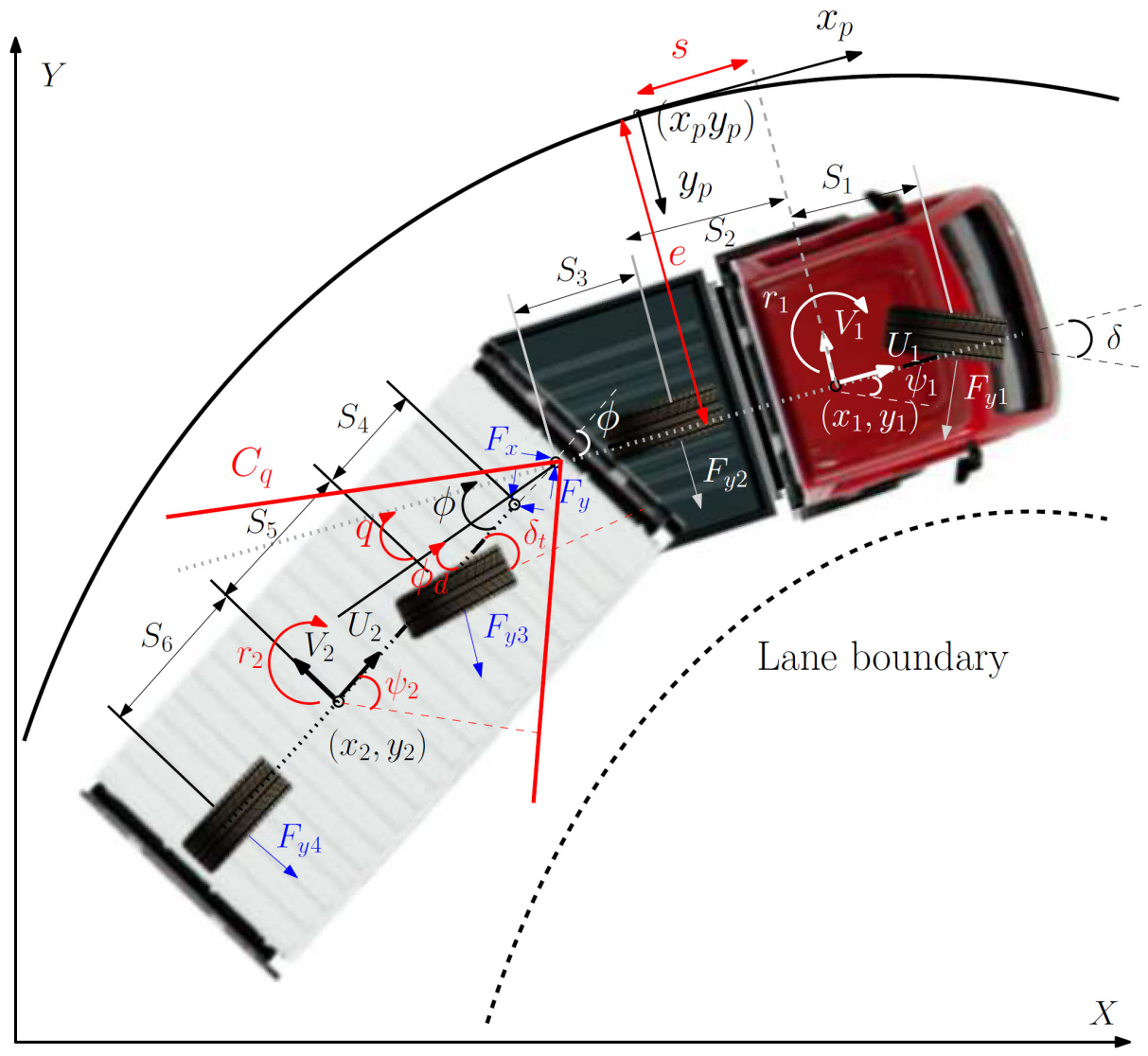


And the trailer's dynamics are parameterised as in Islam et al. (2012):

$$
\begin{aligned}
& \dot{U}_{2}=\frac{1}{m_{2}}\left\{-F_{y 3} \sin \left(\delta_{t}\right)-F_{x} \cos (\phi)-F_{y} \sin (\phi)+V_{2} r_{2}\right\} \\
& \dot{V}_{2}=\frac{1}{m_{2}}\left\{F_{y 4}+F_{y 3} \cos \left(\delta_{t}\right)-F_{x} \sin (\phi)+F_{y} \cos (\phi)-U_{2} r_{2}\right\} \\
& \dot{r}_{2}=\frac{1}{I_{2}}\left\{-F_{y 4} S_{6}+F_{y 3} S_{5} \cos \left(\delta_{t}\right)+F_{y} e \cos (\phi)-F_{x} e \sin (\phi)\right\}
\end{aligned}
$$

where $U_{2}$ and $V_{2}$ present the trailer's velocities, $m_{1}, m_{2}, I_{1}, I_{2}$ denote the truck and trailer mass and inertia respectively. $\delta$ and $\delta_{t}$ are the steering angles of the truck and the trailer, respectively. The articulation angle $\phi$ is equal to the difference between $\psi_{1}$ and $\psi_{2}$. The applied forces are defined as $F_{y 1}=C_{1}\left(\delta-\frac{V_{1}+d r_{1}}{U_{1}}\right), F_{y 2}=C_{2}\left(\frac{V_{1}-d r}{U_{1}}\right)$, $F_{y 3}=C_{3}\left(\delta_{t}-\frac{V_{2}-S_{6} r}{U_{2}}\right)$ and $F_{y 4}=C_{4}\left(\frac{V_{2}-S_{6} r}{U_{2}}\right)$.

In this paper, the RISE feedback controller is applied to control the truck and the trailer's steering angle. For this, the vehicle's dynamic model has to be linearised on the basis of a few assumptions.

Assumption 1: To simplify the adopted vehicle model, one may suppose that the truck's and the trailer's longitudinal velocities $U_{1}$ and $U_{2}$ are constants and equal. As the developed system is designed for highway road, the truck steering angle $\delta$ and the articulation angle $\phi$ are assumed small.

The compact linear model is hence developed in the state space form as:

$$
M \ddot{z}=D \dot{z}+B u
$$

where the matrices $M, D$ and $B$ are expressed in Appendix, the state $z=\left[\int V_{1}, \psi_{1}, \phi, \int \phi\right]^{T}$ and the control input $u=\left[\delta_{t}, \delta\right]^{T}$.

Assumption 2: $M$ is symmetric and positive definite inertia matrix that respects the inequality below, $\forall z \in \mathbb{R}^{4}$

$$
\underline{m}\|z\|^{2} \leq z^{\mathrm{T}} M z \leq \bar{m}\|z\|^{2}
$$

where $\underline{m} \in \mathbb{R}_{+}$and $\bar{m} \in \mathbb{R}_{+}$, are respectively the minimum and maximum eigenvalues of $M$.

\section{Dynamic controller}

The control objective in this paper is to converge the lateral and the longitudinal tracking errors $e$ and $s$ to zero and to converge the articulation angle $\phi$ to the desired 
articulation angle $\phi_{d}$ such that the articulation error $q$ goes to zero. Although the tracking and articulation errors go to zero, some constraints are applied to limit the truck with the trailer's position to stay within the lane boundaries. It is worth to mention that the trailer's position may be monitored using the following relations:

$$
\begin{aligned}
& x_{2}=x_{1}-d \cos \left(\psi_{1}\right)-e \cos \left(\psi_{2}\right) \\
& y_{2}=x_{1}-d \sin \left(\psi_{1}\right)-e \sin \left(\psi_{2}\right)
\end{aligned}
$$

\subsection{Guidance}

As the truck follows a reference trajectory, the longitudinal and the lateral tracking errors are presented as:

$$
\left[\begin{array}{l}
s \\
e
\end{array}\right]=\left[\begin{array}{cc}
\cos \psi_{1 p} & -\sin \psi_{1 p} \\
\sin \psi_{1 p} & \cos \psi_{1 p}
\end{array}\right]^{T}\left[\begin{array}{l}
x_{1}-x_{1 d}(\theta) \\
y_{1}-y_{1 d}(\theta)
\end{array}\right]
$$

where $\psi_{p}=\arctan \left(y_{p}^{\prime}(\theta), x_{p}^{\prime}(\theta)\right)$ and $\left(x_{1 p}(\theta), y_{1 p}(\theta)\right)$ are the trajectory tangential angle and the coordinates of the tracked reference point respectively. From Osman et al. (2019), the expression of the derivative of the tracking errors are established as:

$$
\begin{aligned}
\dot{e} & =v_{1}+v_{1}\left(\cos \left(\tilde{\psi}_{1}\right)-1\right) \\
& +v_{2} \sin \left(\tilde{\psi}_{1}\right)+\tilde{V}_{1} \sin \left(\psi_{1}-\psi_{p 1}\right)-\dot{\psi}_{1 p} s \\
\dot{s} & =e \dot{\psi}_{1 p}+V_{1} \cos \left(\psi_{1}-\psi_{1 p}\right)-\dot{\theta} \sqrt{x_{1 d}^{\prime 2}(\theta)+y_{1 d}^{\prime 2}(\theta)}
\end{aligned}
$$

with $v_{1}$ and $v_{2}$ defined as follows:

$$
\begin{aligned}
& v_{1}=V_{1 d} \sin \left(\psi_{1 d}-\psi_{1 p}\right) \\
& v_{2}=V_{1 d} \cos \left(\psi_{1 d}-\psi_{1 p}\right)
\end{aligned}
$$

$V_{1 d}$ is the desired longitudinal truck velocity. The truck's orientation error is calculated as $\tilde{\psi}_{1}=\psi_{1}-\psi_{1 d}$ and the truck's linear velocity error is expressed as $\tilde{V}_{1}=V_{1}-V_{1 d}$. The derivative of the articulation angle error denotes

$$
\begin{aligned}
\dot{q} & =\dot{\phi}_{-} \dot{\phi}_{d} \\
& =\dot{\tilde{\psi}}_{1}+\dot{\psi}_{1 d}-\dot{\psi}_{2}-\dot{\psi}_{1 d}+\dot{\psi}_{2 d} \\
& =\dot{\tilde{\psi}}_{1}-\dot{\psi}_{2}+\dot{\psi}_{2 d}
\end{aligned}
$$

where $\phi_{d}=\psi_{1 d}-\psi_{2 d}$, the values of $\psi_{1 d}$ and $\psi_{2 d}$ are determined later using the logarithm Barrier Lyapunov Function (BLF). In addition to the applied constraints on the truck's COG position that should follow a desired trajectory, the articulation angle between the truck and the trailer must be limited within a predefined interval which 
indirectly bounds the position of the trailer's COG. The truck-trailer vehicle's position may be bounded by limiting the tracking errors $e$ and $s$, and the articulation error $q$ such as:

$$
|e(t)|<C_{e}, \quad|s(t)|<C_{s}, \quad|q(t)|<C_{q}
$$

Proposition 1: The equilibrium point $z=(e, s, q)^{T}=(0,0,0)^{T}$ is rendered asymptotically stable if the following guidance laws are selected as:

$$
\begin{aligned}
& \dot{\theta}=\frac{V_{1} \cos \left(\psi_{1}-\psi_{1 p}\right)+k_{1} s+\dot{\psi}_{1 p}\left(e-\frac{e_{\log }}{s_{\log }} s\right)}{\sqrt{x_{d}^{\prime 2}(\theta)+y_{d}^{\prime 2}(\theta)}} \\
& \psi_{1 d}= \psi_{1 p}+\arctan \left(\frac{-e}{\Delta}\right) \\
& \psi_{2 d}=\dot{\psi}_{2}-k_{q} q_{\log }
\end{aligned}
$$

where $k_{1}, k_{q}$ and $\Delta$ are positive gains.

Furthermore, if the initial conditions are $z(0) \in\left\{z \in \mathbb{R}^{3}:|z| \leq D_{z}\right\}$, where $D_{z}=\max \left\{C_{e}, C_{s}, C_{q}\right\} \sqrt{1-e^{-2 V_{t}(0)}}$, then the constraints are satisfied. Hence, the guidance system is Locally Exponentially Stable (LES).

To achieve this task, the BLF is used as:

$$
V_{t}=\frac{1}{2} \log \frac{C_{s}^{2}}{C_{s}^{2}-s^{2}}+\frac{1}{2} \log \frac{C_{e}^{2}}{C_{e}^{2}-e^{2}}+\frac{1}{2} \log \frac{C_{q}^{2}}{C_{q}^{2}-q^{2}}
$$

Hence the derivative of the Lyapunov function is calculated as:

$$
\begin{aligned}
\dot{V}_{t} & =\frac{s \dot{s}}{C_{s}^{2}-s^{2}}+\frac{e \dot{e}}{C_{e}^{2}-e^{2}}+\frac{q \dot{q}}{C_{q}^{2}-q^{2}} \\
& =s_{\log }\left(e \dot{\psi} \dot{1 p}_{p}+v \cos \left(\psi_{1}-\psi_{1 p}\right)\right. \\
& -\dot{\theta} \sqrt{x_{1 d}^{2}(\theta)+y_{1 d}^{2}(\theta)}+e_{\log }\left(v_{1}+v_{1}\left(\cos \left(\tilde{\psi}_{1}\right)\right)-1\right) \\
& \left.+v_{2} \sin \left(\tilde{\psi}_{1}\right)+\tilde{V}_{1} \sin \left(\psi_{1}-\psi_{p 1}\right)-\dot{\psi}_{1 p} s\right) \\
& +q_{\log }\left(\dot{\tilde{\psi}}_{1}-\dot{\psi}_{2}+\psi_{2 d}\right)
\end{aligned}
$$

where $s_{\log }=\frac{s}{C_{s}^{2}-s^{2}}, \quad e_{\log }=\frac{e}{C_{e}^{2}-e^{2}}$ and $q_{\log }=\frac{q}{C_{q}^{2}-q^{2}}$ From (12), we confirm the selection of the update laws (10) in Proposition 1. Replacing $\theta, \psi_{1 d}$ and $\psi_{2 d}$ with their functions leads to the following expression: 


$$
\begin{aligned}
\dot{V}_{t}= & -k_{1} s_{\log } s-\frac{V_{1 d} e_{\log } e}{\sqrt{e^{2}+\Delta^{2}}}+e_{\log }\left(-\frac{V_{1 d} e}{\sqrt{e^{2}+\Delta^{2}}}\right. \\
& -\frac{V_{1 d} e}{\sqrt{e^{2}+\Delta^{2}}}\left(\cos \left(\tilde{\psi}_{1}\right)-1\right)+\frac{V_{1 d} \Delta}{\sqrt{e^{2}+\Delta^{2}}} \sin \left(\tilde{\psi}_{1}\right) \\
& \left.+\tilde{V}_{1} \sin \left(\psi_{1}-\psi_{1 p}\right)\right)-k_{q} q_{\log }^{2}+\dot{\tilde{\psi}}_{1} q_{\log }
\end{aligned}
$$

As $\lim _{t \rightarrow \infty} \tilde{\psi}_{1}=0$ and $\lim _{t \rightarrow \infty} \tilde{\psi}_{2}=0$, one may assume that $\lim _{\tilde{\psi}_{1} \rightarrow 0} \frac{\sin \left(\tilde{\psi}_{1}\right)}{\tilde{\psi}_{1}}=1, \lim _{\tilde{\psi}_{1} \rightarrow 0} \frac{\cos \left(\tilde{\psi}_{1}\right)-1}{\tilde{\psi}_{1}}=0$. It follows that $V_{t}(t) \leq V(0) \forall|z(0)| \leq \max \left\{C_{e}, C_{s}, C_{q}\right\} \forall t>0$. Thus, we infer that:

$$
\begin{aligned}
& 0.5 \log \left(C_{s}^{2} / C_{s}^{2}-s^{2}\right) \leq V_{t}(0) \\
& 0.5 \log \left(C_{e}^{2} / C_{e}^{2}-e^{2}\right) \leq V_{t}(0) \\
& 0.5 \log \left(C_{q}^{2} / C_{q}^{2}-q^{2}\right) \leq V_{t}(0)
\end{aligned}
$$

Taking exponentials on both sides of all the inequalities and rearranging, we ultimately obtain that $|z| \leq \max \left\{C_{e}, C_{s}, C_{q}\right\} \sqrt{1-e^{-2 V_{t}(0)}}$.

\subsection{RISE feedback controller}

Based on the compact dynamic trailed truck model, one may define the auxiliary error as:

$$
\begin{aligned}
& \zeta=\dot{z}_{e}+\alpha_{1} z_{e} \\
& \xi=\dot{\zeta}+\alpha_{2} \zeta
\end{aligned}
$$

where $\alpha_{1}$ and $\alpha_{2}$ are positive constants. $z_{e}=\left[\int \tilde{V}_{1}, \tilde{\psi}_{1}, \tilde{\phi}, \int \tilde{\phi}\right]^{T}, \tilde{V}_{1}=V_{1}-V_{1 d}$.

$$
\begin{aligned}
M \xi & =M\left(\dot{\zeta}+\alpha_{2} \zeta\right) \\
& =M\left(\ddot{z}_{e}+\alpha_{1} \dot{z}_{e}+\alpha_{2} \zeta\right) \\
& =M\left(\ddot{z}_{e}+\alpha_{1} \dot{z}_{e}+\alpha_{2}\left(\dot{z}_{e}+\alpha_{1} z_{e}\right)\right) \\
& =Q+S+t
\end{aligned}
$$

where $Q=M \ddot{z}_{d}$ while $S=-D(z, \dot{z})+M\left(\alpha_{1} \dot{z}_{e}+\alpha_{2} \zeta\right)$. However

$$
\begin{aligned}
t & =B u \\
& =-\left(k_{s}+1\right) \zeta(t)+\left(k_{s}+1\right) \zeta(0) \\
& -\int_{0}^{t}\left(k_{s}+1\right) \alpha_{2} \zeta(\sigma)+\rho \operatorname{sgn}(\zeta(\sigma)) d \sigma
\end{aligned}
$$


The closed-loop system is therefore expressed as:

$$
\begin{aligned}
& M \dot{\xi}=\tilde{A}+A_{d}-\zeta-\left(k_{s}+1\right) \xi-\rho \operatorname{sgn}(z) \\
& \tilde{A}=\dot{S}+\zeta \quad \text { and } A_{d}=\dot{Q}
\end{aligned}
$$

From the Mean Value Theorem in Osman et al. (2019), $\tilde{A}$ can be upper limited:

$$
\|\tilde{A}\| \leq \rho(\|\chi\|)\|\chi\|
$$

where $\chi=\left[z_{e}^{\mathrm{T}}, \zeta^{\mathrm{T}}, \xi^{\mathrm{T}}\right]^{\mathrm{T}} \in \mathbb{R}^{3}$ and $\rho() \geq$.0 is invertible function. Also by assuming that the uncertain vector $D(z, \dot{z})$ is bounded, the following inequalities are proved:

$$
\left\|A_{d}\right\| \leq \varsigma_{1}, \quad\left\|\dot{A}_{d}\right\| \leq \varsigma_{2}
$$

where $\varsigma_{1}, \varsigma_{2}$ are positive constants. The controller in (17) confirms the restriction applied on the system signals and ensures the convergence of the pursuit error $z_{e}$ to zero when $t$ goes to infinity. The controller gains are defined according to the following conditions (Osman et al., 2019) in a way that the control system is asymptotically stable.

$$
\alpha_{1}>\frac{1}{2}, \quad \alpha_{2}>\frac{1}{2}, \quad \rho>\left\|A_{d}\right\|_{\infty}+\left\|\dot{A}_{d}\right\|_{\infty}
$$

Theorem 1: Given a smooth reference trajectory $p_{d}(\theta)=\left(x_{d}(\theta), y_{d}(\theta)\right)$ and $\psi_{d}$. The origin of the tracking error $\left(s, e, q, z_{e}, \zeta, \xi\right)$ becomes asymptotically stable by choosing the guidance and the control laws as in (9) and (18) respectively.

The analysis is carried out by viewing the closed-loop system made of the guidance kinematics and the dynamic subsystem as a cascaded interconnection of two subsystems as shown in the following two equations:

$$
\begin{gathered}
\Sigma_{1}\left[\begin{array}{c}
\dot{s} \\
\dot{e} \\
\dot{q}
\end{array}\right]=f(s, e, q)+\underbrace{\left[\begin{array}{ll}
0 & \Psi^{\mathrm{T}}
\end{array}\right]\left[\begin{array}{c}
0 \\
\bar{z}_{e}
\end{array}\right]}_{\Phi\left(s, e, q, \bar{z}_{e}\right)} \\
\Sigma_{2}\left\{\begin{array}{c}
\dot{z}_{e}=\zeta-\alpha_{1} z_{e} \\
\dot{\zeta}=\xi-\alpha_{2} \zeta \\
M \dot{\xi}=\tilde{A}+A_{d}-\zeta-\left(k_{s}+1\right) \xi-\rho \operatorname{sgn}(\zeta)
\end{array}\right.
\end{gathered}
$$

where $f(s, e, q)=\left[-k_{1} s+e \dot{\psi}_{p}-\kappa(s, e) \dot{\psi}_{p},-\frac{v_{d} e}{\sqrt{e^{2}+\Delta^{2}}} r,-k_{q} q_{\log }+\dot{\tilde{\psi}}_{2}\right]^{\mathrm{T}}$ and $\Psi=\left[\sin \left(\psi-\beta-\psi_{p}\right)\right.$, $\left.-\frac{v_{d} e}{\sqrt{e^{2}+\Delta^{2}}}\left(\frac{\cos (\tilde{\psi})-1}{\tilde{\psi}}\right)+\frac{v_{d} \Delta}{\sqrt{e^{2}+\Delta^{2}}} \frac{\sin (\tilde{\psi})}{\tilde{\psi}}, 1,0\right]$. In this regard, since we showed that 
$\Sigma_{2}$ is asymptotically stable, by showing that $\Sigma_{1}$ is Input-to-State Stable (ISS) with respect to $\bar{z}_{e}$. Thus the whole system is asymptotically stable.

\section{Numerical simulation}

In this section, the efficiency of the developed approach is validated for lane-keeping scenario. The vehicle model under study is simulated by introducing some uncertainties on its parameters. For sake of clarity, random numbers are added to the vehicle's mass and inertia as follows: $m_{1}(\mathrm{~kg})=6825+100 \mathrm{rand}(),. m_{2}(\mathrm{~kg})=5460+100 \mathrm{rand}($.$) ,$ $I_{1}=21.693+\operatorname{rand}($.$) and I_{2}=12.540+\operatorname{rand}($.$) , where \operatorname{rand}($.$) presents a random$ number between 0 and 1 . The vehicle's length and the wheels' cornering stiffness are parameterised as in Osman et al. (2019). The chosen gains for the proposed controller are $\Delta=0.2, k_{2}=18, \alpha_{1}=3, \alpha_{2}=5$ and $k_{s}=4.5$; The applied constraints on the longitudinal and lateral position tracking are presented respectively by $C e=10$ and $C s=3$. Otherwise, $C q=2$ denotes the articulation angle error bound.

Multiple road scenarios are illustrated to validate the proposed system. Figure 3 shows a straight lane-keeping scenario. The truck's and the trailer's COG $x y_{1}$ and $x y_{2}$ are following the desired truck and trailer trajectory $x y_{1 d}$ and $x y_{2 d}$, respectively. The lateral and longitudinal truck's position errors and the articulation angle error are converging to zero in Figure 4. It is shown in Figure 5 that the truck and its trailer are following the desired circular trajectory. We notice a small gap between the desired and the travelled trajectories in this circular scenario due to the neglecting of the sideslip angle in the vehicle mathematical modelling. This gap is clearer in this scenario w.r.t the straight lane case because of the increased path curvature. Since the circular trajectory is based on a periodic signal, the tracking and articulation errors are converging to zero with a periodic peak reflecting the starting point of the desired path in Figure 6 . The following scenario concerns a curved road. Even by starting from different initial conditions, the truck and trailer's positions follow the desired trajectory. From Figure 7, one may notice that the trailer position follows the truck position but with small time gap due to the distance separating the trailer's COG from the truck's COG. In addition, the desired orientation is obviously followed by the vehicle's heading angles $\psi_{1}$ and $\psi_{2}$ in Figure 8. The control inputs $\delta$ and $\delta_{t}$ allowing the desired path following are presented in Figure 9. A comparison study with the Computed Torque Controller (CTC) is thereafter implemented. In fact, the CTC torque expression is defined as follows:

$$
\tau=M\left(\ddot{z}_{d}+K_{d} \dot{z}_{e}+K_{p} z_{e}\right)-D \dot{z}
$$

where $K_{p}$ and $K_{d}$ are $4 X 4$ diagonal positives matrices. 
Figure 3 Truck and trailer position in a straight lane case

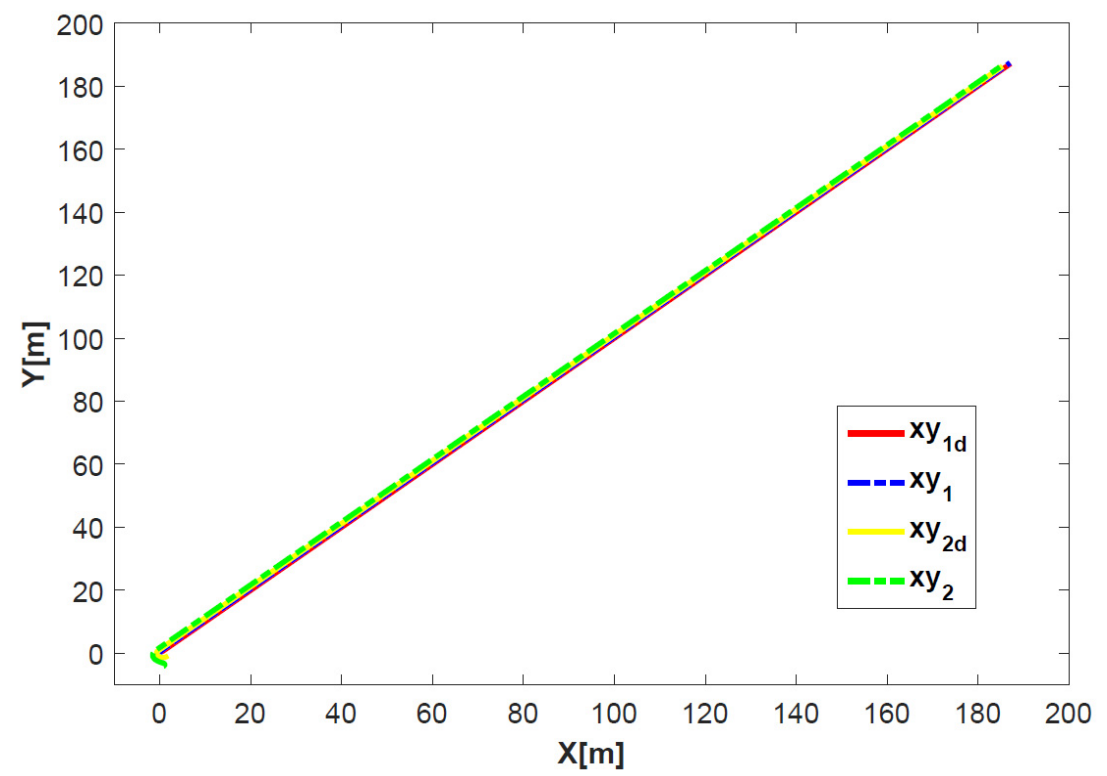

Figure 4 Tracking and articulation errors in a straight lane case
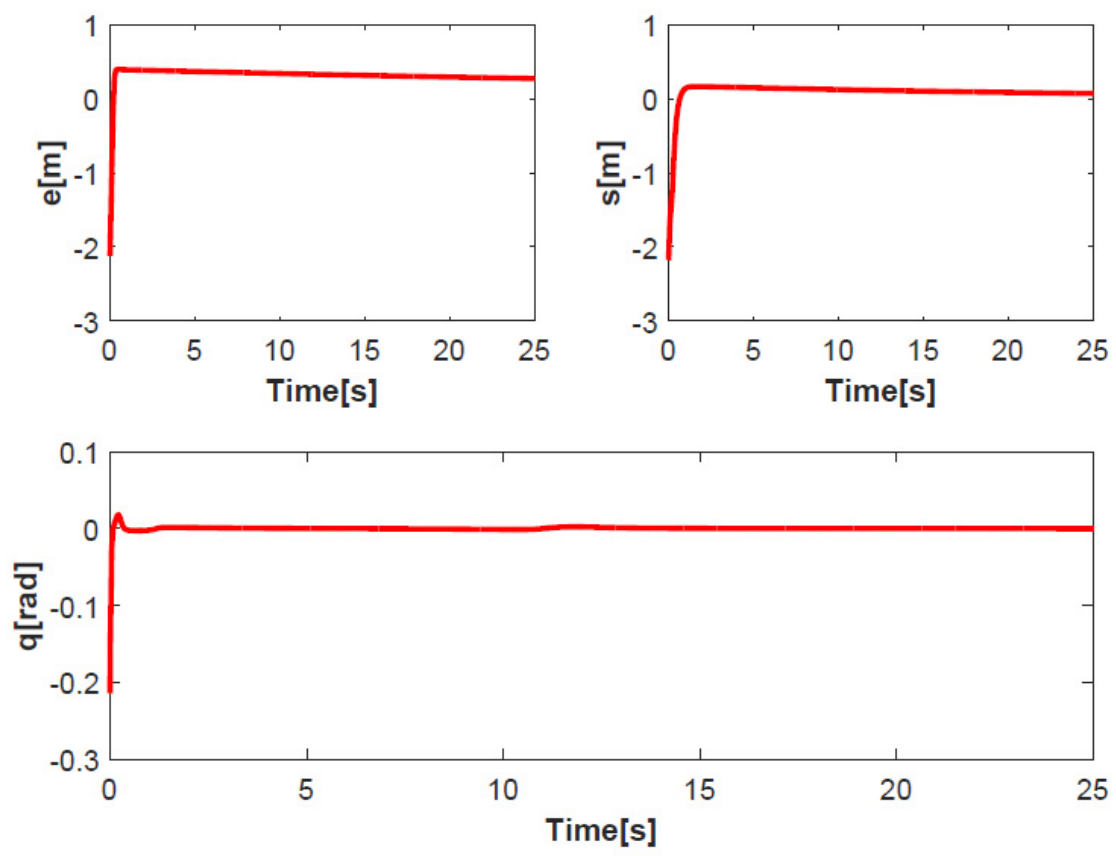
Figure 5 Truck and trailer position in a circular lane case

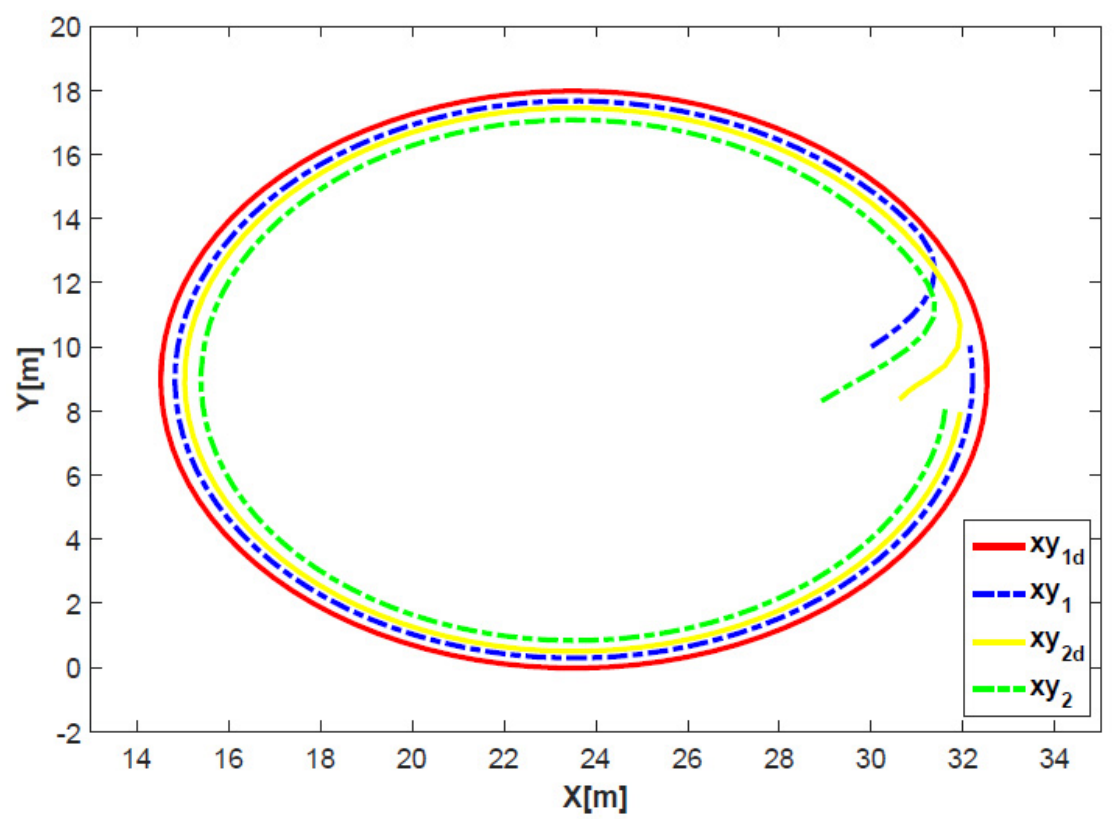

Figure 6 Tracking and articulation errors in a circular lane case
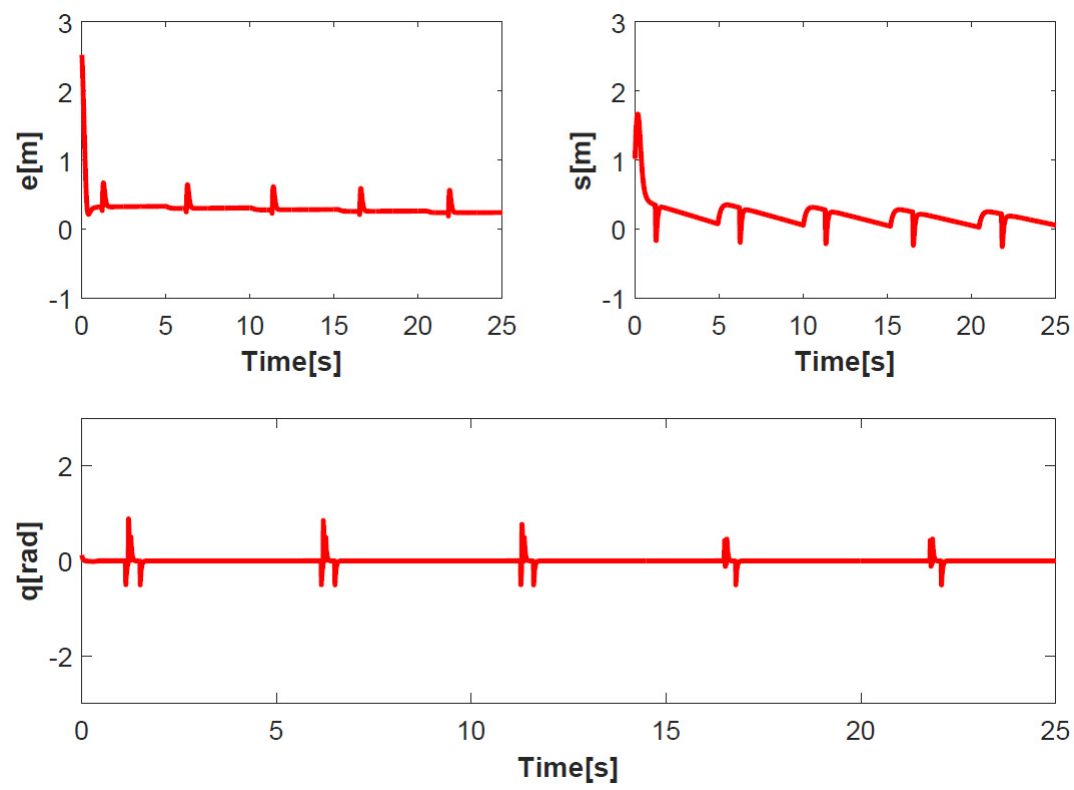
Active steering control-based trucks lane keeping system

Figure 7 Truck and trailer position in a curved lane case

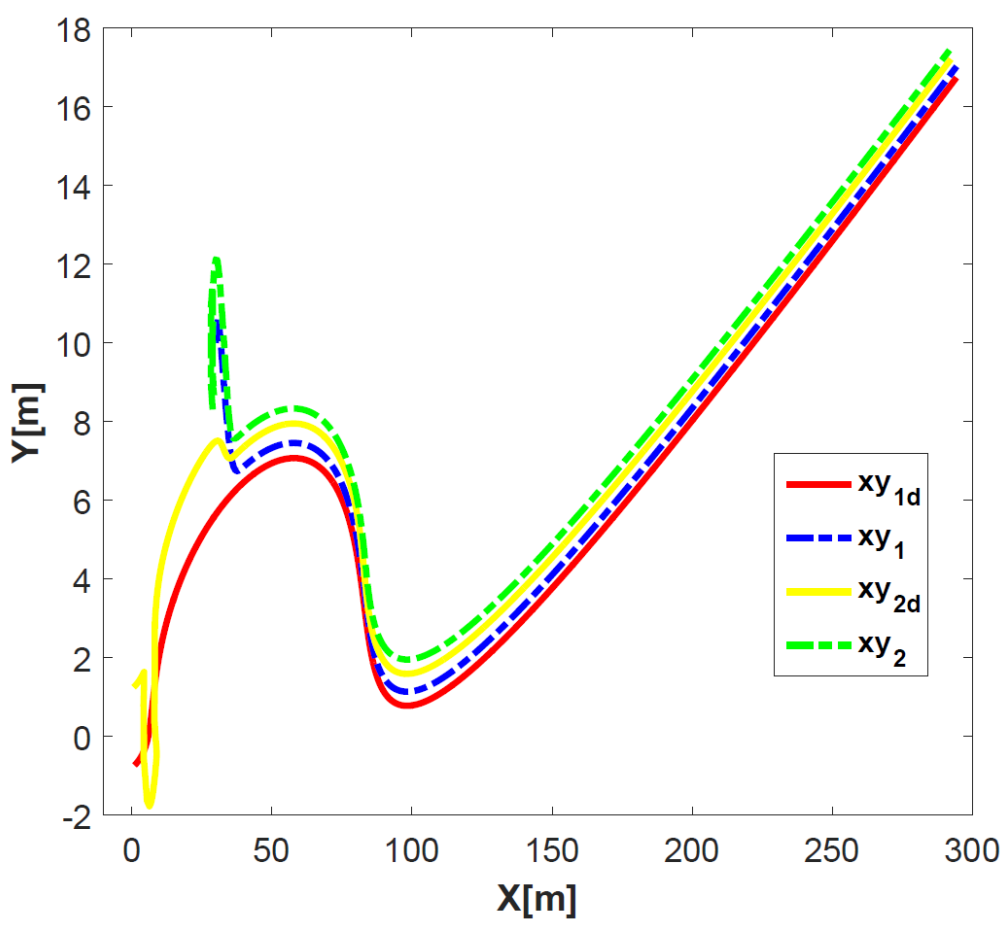

Figure 8 Truck and trailer's heading angle in a curved lane case
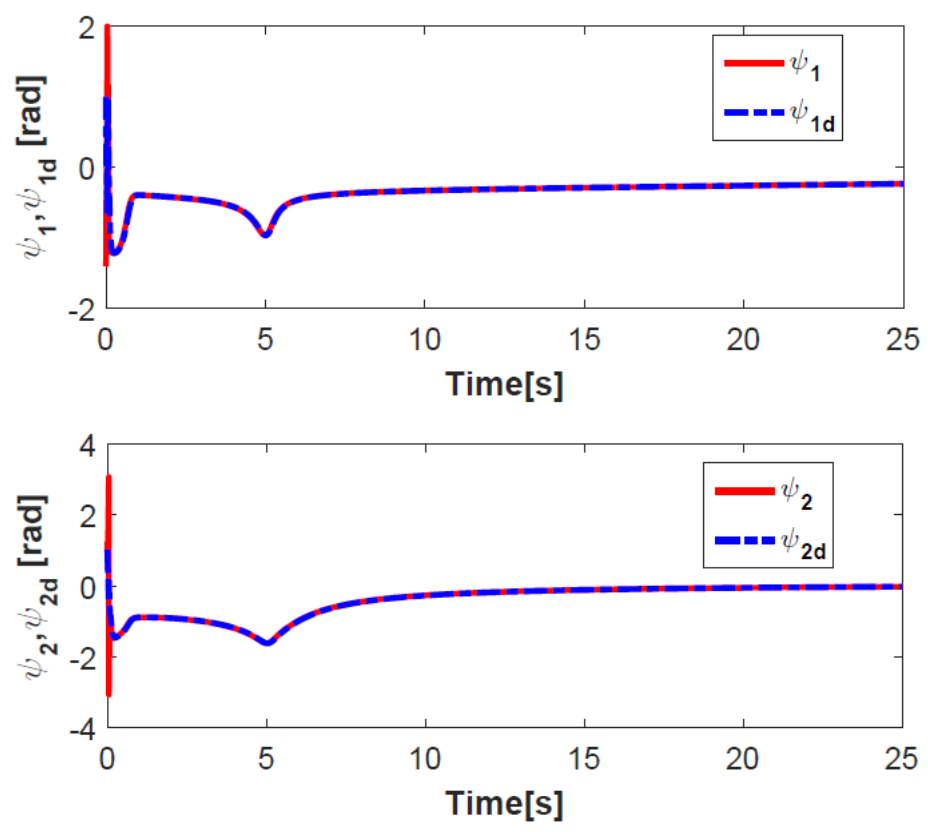
Figure 9 Control inputs in a curved lane case
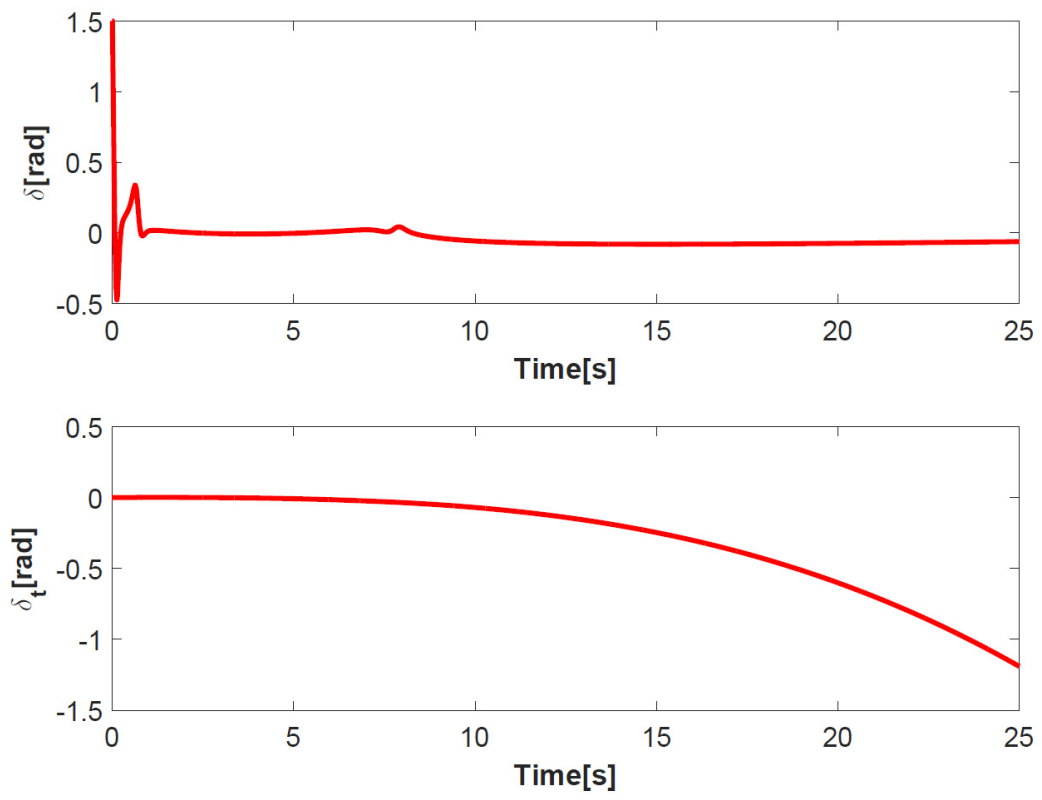

Figure 10 A comparison between the RISE and CTC tracking errors in a curved lane case
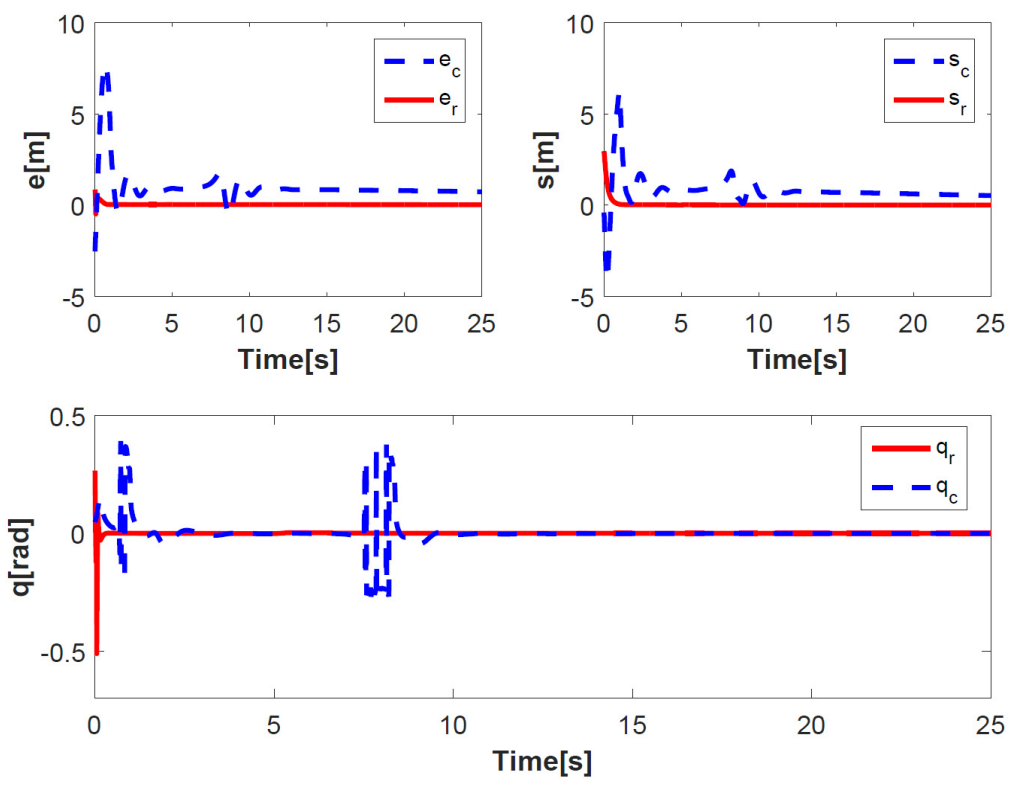
It is noticed that the lateral $s_{r}$, the longitudinal $e_{r}$ and the articulation angle $q_{r}$ errors are converging to zero. Also, the predefined errors constraints $C_{e}, C_{s}$ and $C_{q}$ are respected as illustrated in Figure 10. Otherwise, it is shown that the tracking errors $s_{c}$, $e_{c}$ and the articulation error $q_{c}$ of the CTC exceed the aforementioned bounds and present an oscillatory behaviour due the model parameter uncertainties.

\section{Conclusion}

In this paper, an active trailer steering model is used to simulate the articulated vehicle's dynamic in the lane-keeping scenario. A LOS guidance algorithm is developed to limit the articulated vehicle's position inside the lane boundaries. The Barrier Lyapunov function is thus used to impose constraints on the truck position and the articulation angle errors. In order to follow the desired constrained trajectory, a RISE feedback controller is designed while compensating the vehicle model's uncertainties. A numerical simulation is finally illustrated to prove the efficiency of the developed approach in various scenarios. In addition, a comparative study with the computed torque controller is presented. In future work, the sideslip angle will be considered in modelling the articulated vehicle.

\section{References}

Chen, C. and Tomizuka, M. (2000) 'Lateral control of commercial heavy vehicles', Vehicle System Dynamics, Vol. 33, No. 6, pp.391-420.

Chen, F., Song, M., Ma, X. and Zhu, X. (2019) 'Assess the impacts of different autonomous trucks lateral control modes on asphalt pavement performance', Transportation Research Part C: Emerging Technologies, Vol. 103, pp.17-29.

Hac, A., Fulk, D. and Chen, H. (2008) 'Stability and control considerations of vehicle-trailer combination', SAE International Journal of Passenger Cars-Mechanical Systems, pp.925-937.

Islam, M.M., Ding, X. and He, Y. (2012) 'A closed-loop dynamic simulation-based design method for articulated heavy vehicles with active trailer steering systems', Vehicle System Dynamics, Vol. 50, No. 5, pp.675-697.

Kassaeiyan, P., Tarvirdizadeh, B. and Alipour, K. (2019) 'Control of tractor-trailer wheeled robots considering self-collision effect and actuator saturation limitations', Mechanical Systems and Signal Processing, Vol. 127, pp.388-411.

Keymasi-Khalaji, A., Rahimi-Bidgoli, M. and Moosavian, S.A.A. (2015) 'Non-model-based control for a wheeled mobile robot towing two trailers', Proceedings of the Institution of Mechanical Engineers, Part K: Journal of Multi-body Dynamics, Vol. 229, No. 1, pp.97-108.

Khalaji, A.K. (2019) 'Modeling and control of uncertain multibody wheeled robots', Multibody System Dynamics, Vol. 46, No. 3, pp.257-279.

Liu, Y., Ji, X., Yang, K., He, X., Na, X. and Liu, Y. (2020) 'Finite-time optimized robust control with adaptive state estimation algorithm for autonomous heavy vehicle', Mechanical Systems and Signal Processing, Vol. 139. Doi: 10.1016/j.ymssp.2020.106616.

Miao, Q. and Cebon, D. (2019) 'Path-tracking errors for active trailer steering off-highway: a simulation study', International Journal of Heavy Vehicle Systems, Vol. 26, Nos. 3/4. Doi: 10.1504/IJHVS.2019.10022768. 
Montiglio, M., Martini, S. and Murdocco, V. (2006) 'Development of a lane keeping support system for heavy trucks', Proceedings of the 13th ITS World Congress, pp.1-9.

Nilsson, J. (2013) U.S. Patent No. 8,428,821, U.S. Patent and Trademark Office, Washington, DC.

Osman, K., Ghommam, J., Mehrjerdi, H. and Saad, M. (2019) 'Vision-based curved lane keeping control for intelligent vehicle highway system', Proceedings of the Institution of Mechanical Engineers, Part I: Journal of Systems and Control Engineering, Vol. 233, No. 8, pp.961-979.

Pillajo-Quijia, G., Arenas-Ramrez, B., Gonzlez-Fernndez, C. and Aparicio-Izquierdo, F. (2020) 'Influential factors on injury severity for drivers of light trucks and vans with machine learning methods', Sustainability, Vol. 12, No. 4, pp.1-29.

Qingyun, W., Peng, W. and Ning, C. (2017) 'Dynamic modeling and simulation of trailer with compliance steering system', Proceedings of the 3rd International Conference on Mechanical, Electronic and Information Technology Engineering, EDP Sciences, Vol. 139, pp.1-5.

Sename, O., Dugard, L. and Gaspar, P. (2019) 'Hळ/LPV controller design for an active anti-roll bar system of heavy vehicles using parameter dependent weighting functions', Heliyon, Vol. 5, No. 6, pp.1-11.

Shamim, R., Islam, M.M. and He, Y. (2011) A Comparative Study of Active Control Strategies for Improving Lateral Stability of Car-Trailer Systems (No. 2011-01-0959), SAE Technical Paper.

Shi, Y., Chen, Y. and Jia, B. (2019) 'Local trajectory planning for autonomous trucks in collision avoidance maneuvers with rollover prevention', Proceedings of the American Control Conference (ACC), IEEE, pp.3981-3986.

$\mathrm{Xu}, \mathrm{X}$., Zhang, L., Jiang, Y. and Chen, N. (2019) 'Active control on path following and lateral stability for truck trailer combinations', Arabian Journal for Science and Engineering, Vol. 44, No. 2, pp.1365-1377.

Yue, M., Hou, X., Gao, J. and Yang, L. (2019) 'RBFNN based identification and compensation mechanism for disturbance like parametric friction with application to tractor trailer vehicles', Asian Journal of Control, Vol. 22, No. 1, pp.398-410.

Zhang, B., Zong, C., Chen, G., Huang, Y. and Xu, T. (2019) 'A novel integrated stability control based on differential braking and active steering for four-axle trucks', Chinese Journal of Mechanical Engineering, Vol. 32, No. 12, pp.1-21.

Zhang, J., Wu, K., Cheng, M., Yang, M., Cheng, Y. and Li, S. (2020) 'Safety evaluation for connected and autonomous vehicles exclusive lanes considering penetrate ratios and impact of trucks using surrogate safety measures', Journal of Advanced Transportation, pp.1-16.

Zhu, S., He, Y. and Ren, J. (2019) 'On robust controllers for active steering systems of articulated heavy vehicles', International Journal of Heavy Vehicle Systems, Vol. 26, No. 1, pp.1-30. 


\section{Appendix}

$$
\begin{aligned}
M & =\left[\begin{array}{cccc}
m_{1}+m_{2} & -m_{2} d-m_{2} e & m_{2} d+m_{2} e & 0 \\
-m_{2} d & m_{2} d(d+e)+I_{1} & -m_{2} d(d+e) & 0 \\
-m_{2} e & m_{2} e(d+e)+I_{2} & -m_{2} e(d+e)-I_{2} & 0 \\
0 & 0 & 0 & 1
\end{array}\right] \\
D & =\left[\begin{array}{cccc}
d_{11} & d_{12} & d_{13} & d_{14} \\
d_{21} & d_{22} & d_{23} & d_{24} \\
d_{31} & d_{32} & d_{33} & d_{34} \\
0 & 0 & 1 & 0
\end{array}\right] \\
B & =\left[\begin{array}{ccc}
-C_{1} & -C_{3} \\
-C_{1} S_{1} & -C_{3} d \\
0 & C_{3}\left(e-S_{5}\right) \\
0 & 0
\end{array}\right]
\end{aligned}
$$

$d=S_{2}+S_{3}$ and $e=S_{4}+S_{5}$

$d_{11}=\frac{1}{U_{1}}\left\{C_{1}+C_{2}+C_{3}+C_{4}\right\}$

$d_{12}=\frac{1}{U_{1}}\left\{C_{1} S_{1} C_{2} S_{2} C_{3}\left(d+e S_{5}\right) C_{4}\left(d+e+S_{6}\right)\right\}\left(m_{1}+m_{2}\right) U_{1}$

$d_{13}=\frac{1}{U_{1}}\left\{C_{3}\left(d+e S_{5}\right)+C 4\left(d+e+S_{6}\right)\right\}$

$d_{14}=C_{3}+C_{4}$

$d_{21}=\frac{1}{U_{1}}\left\{C_{1} S_{1} C_{2} S_{2} C_{3} d C_{4} d\right\}$

$d_{22}=\frac{1}{U_{1}}\left\{C_{1} S_{1}^{2}+C_{2} S_{2}^{2}+C_{3} d\left(d+e S_{5}\right)+C_{4} d\left(d+e S_{6}\right)\right\}+m_{2} d U_{1}$

$d_{23}=\frac{1}{U_{1}}\left\{C_{3} d\left(d+e S_{5}\right)+C_{4} d\left(d+e S_{6}\right)\right\}$

$d_{24}=d\left(C_{3}+C_{4}\right)$

$d_{31}=\frac{1}{U_{1}}\left\{C_{3}\left(e S_{5}\right)+C_{4}\left(e+S_{6}\right)\right\}$

$d_{32}=\frac{1}{U_{1}}\left\{C_{3}\left(e S_{5}\right)\left(d+e S_{5}\right)+C_{4}\left(e+S_{6}\right)\left(d+e+S_{6}\right)\right\}+m_{2} e U_{1}$

$d_{33}=\frac{1}{U_{1}}\left\{C_{3}\left(e S_{5}\right)\left(d+e S_{5}\right)+C_{4}\left(e+S_{6}\right)\left(d+e+S_{6}\right)\right\}$

$d_{34}=C_{3}\left(e S_{6}\right) C_{4}\left(e+S_{6}\right)$ 